\title{
ON A PROBLEM OF TURÁN ABOUT POLYNOMIALS
}

\author{
R. PIERRE AND Q. I. RAHMAN 1
}

\begin{abstract}
It is shown that if $p_{n}(x)$ is a polynomial of degree $n$ whose graph on the interval $-1<x<1$ is contained in the unit disk then the absolute value of its second derivative cannot exceed $\frac{2}{3}(n-1)\left(2 n^{2}-4 n+3\right)$ on $[-1,1]$.
\end{abstract}

In the year $1889 \mathrm{~A}$. A. Markoff [2] proved the following result:

THEOREM A. If $p_{n}(x)=\sum_{\nu=0}^{n} a_{\nu} x^{\nu}$ is a polynomial of degree $n$ and $\left|p_{n}(x)\right|$ $\leq 1$ in the interval $-1 \leq x \leq 1$ then in the same interval

$$
\left|p_{n}^{\prime}(x)\right| \leq n^{2} .
$$

The constant $n^{2}$ in (1) cannot be replaced by any lower constant. In fact, the $n$th Tchebycheff polynomial of the first kind

$$
T_{n}(x)=\cos (n \operatorname{arc} \cos x)=2^{n-1} \prod_{\nu=1}^{n}\left\{x-\cos \left(\left(\nu-\frac{1}{2}\right) \pi / n\right)\right\}
$$

satisfies the conditions of Theorem A and its derivative at the point $x=1$ is equal to $n^{2}$.

W. A. Markoff (brother of A. A. Markoff) considered the problem of determining exact bounds for the $k$ th derivative of $p_{n}(x)$ at a given point $x_{0}$ in $[-1,1]$ under the conditions of Theorem A. His results appeared in a Russian journal in the year 1892; a German version of his remarkable paper was later published in Mathematische Annalen [3]. Amongst other things he proved:

THEOREM B. Under the conditions of Theorem A

$$
\begin{aligned}
& \max _{-1 \leq x \leq 1}\left|p_{n}^{(k)}(x)\right| \leq \frac{n^{2}\left(n^{2}-1^{2}\right)\left(n^{2}-2^{2}\right) \cdots\left(n^{2}-(k-1)^{2}\right)}{1 \cdot 3 \cdot 5 \cdots(2 k-1)}, \\
& k=1,2, \ldots, n .
\end{aligned}
$$

The right-hand side of this inequality is exactly equal to $T_{n}^{(k)}(1)$, where $T_{n}(x)$ is the $n$th Tchebycheff polynomial of the first kind (2). W. A. Markoff's proof

Presented to the Society, October 27, 1973 under the title On a problem of Turán; received by the editors March 31, 1975.

AMS (MOS) subject classifications (1970). Primary 30A06, 30A40; Secondary 42A04.

Key words and phrases. Polynomials with curved majorants, Tchebycheff polynomial of the first kind, Tchebycheff polynomial of the second kind.

${ }^{1}$ This work was supported by National Research Council of Canada Grant A-3081 and by a grant of le Ministère de l'Education du Gouvernement du Québec.

- American Mathematical Society 1976 
of this result is based on a method which applies only to polynomials with real coefficients, but this is a restriction which is easily removed. For if $p_{n}(x)$ is any polynomial which satisfies the conditions of the theorem and $\gamma$ is a complex constant of unit modulus then $\gamma p_{n}(x)$ will satisfy the conditions of the theorem and so will $\operatorname{Re}\left\{\gamma p_{n}(x)\right\}$. But $\gamma$ can be so chosen that the derivative of $\gamma p_{n}(x)$ at any preassigned point is real, and then the derivative of $\operatorname{Re}\left\{\gamma p_{n}(x)\right\}$ will have the same modulus as $p_{n}^{\prime}(x)$ at this point.

For fixed $k$ suppose that $p_{n}(x)$ is an extremal polynomial, that is, suppose its $k$ th derivative assumes the maximum possible value under the conditions of the theorem. (It is easily shown that such polynomials exist.) Markoff used a variational method to show that $\left|p_{n}(x)\right|$ must be equal to 1 at either $n$ or $n+1$ different points in the interval $[-1,1]$. In the latter case $\pm p_{n}(x)$ is the $n$th Tchebycheff polynomial of the first kind, whose derivatives are easily shown to satisfy (3). In the former case it is possible to show that $p_{n}(x)$ satisfies a differential equation of the form

$$
1-\left(p_{n}(x)\right)^{2}=\frac{\left(1-x^{2}\right)(x-b)(x-c)}{n^{2}(x-a)^{2}}\left(p_{n}^{\prime}(x)\right)^{2} .
$$

Here $a, b, c$ are real constants which depend upon one parameter. Markoff was then able to show that the derivatives of this class of polynomials satisfy (3), but the proof is quite difficult.

W. A. Markoff [3] started out by taking a very general point of view: If $\lambda_{0}, \lambda_{1}, \ldots, \lambda_{n}$ are given constants and $p_{n}(x)=\sum_{\nu=0}^{n} a_{\nu} x^{\nu}$ satisfies the conditions of the theorem, what is the precise bound for the linear form $\sum_{\nu=0}^{n} a_{\nu} \lambda_{\nu}$ ? By suitably choosing the constants $\lambda_{\nu}$ the linear form can be made equal to any derivative of $p_{n}(x)$ at any preassigned point.

For polynomials with real coefficients the hypothesis $\left|p_{n}(x)\right| \leq 1$ for $-1 \leq x \leq 1$ means that the graph of $p_{n}(x)$ on the interval $-1 \leq x \leq 1$ lies in the square $\left\{(x, y) \in R^{2}:-1 \leq x \leq 1,-1 \leq y \leq 1\right\}$. Recently Professor P. Turán proposed consideration of polynomials whose graph on the unit interval lies in the unit disk. Such type of problems occured first in the approximation theory, notably in the work of Dzjadik on converse type theorems concerning approximation by rational polynomials in $[-1,+1]$.

One of us has proved [5] the following analogue of Theorem A.

THEOREM C. If $p_{n}(x)$ is a polynomial of degree $n$ such that $\left|p_{n}(x)\right|$ $\leq\left(1-x^{2}\right)^{\frac{1}{2}}$ for $-1 \leq x \leq 1$, then

$$
\max _{-1 \leq x \leq 1}\left|p_{n}^{\prime}(x)\right| \leq 2(n-1) \text {. }
$$

If

$$
U_{n}(x)=\left(1-x^{2}\right)^{-\frac{1}{2}} \sin \{(n+1) \operatorname{arc} \cos x\}
$$

is the nth Tchebycheff polynomial of the second kind then $p_{n}(x)=$ $\left(1-x^{2}\right) U_{n-2}(x)$ satisfies the conditions of Theorem $\mathrm{C}$ and $\left|p_{n}^{\prime}( \pm 1)\right|=2(n-1)$.

Here we consider the problem of estimating $\max _{-1 \leq x \leq 1}\left|p_{n}^{(k)}(x)\right|$ under the conditions of Theorem $\mathrm{C}$. Although we have not been able to solve the 
problem completely, we do show that

$$
\max _{-1 \leq x \leq 1}\left|p_{n}^{\prime \prime}(x)\right| \leq\left|\left(d^{2} / d x^{2}\right)\left(\left(1-x^{2}\right) U_{n-2}(x)\right)\right|_{x= \pm 1} .
$$

We are tempted to conjecture that

$$
\max _{-1 \leq x \leq 1}\left|p_{n}^{(k)}(x)\right| \leq\left|\left(d^{k} / d x^{k}\right)\left(\left(1-x^{2}\right) U_{n-2}(x)\right)\right|_{x= \pm 1}
$$

holds also for $k=3,4, \ldots, n$.

We observe that many of the ideas of W. A. Markoff when slightly modified apply to the present problem. However, part of his reasoning is very hard to carry over. It was only in the case $k=2$ that we could get around the difficulties by using an idea which has been previously used by $\mathbf{S}$. N. Bernstein, B. Ya. Levin and others.

Consider the real linear space $\mathscr{P}_{n}$ of all polynomials

$$
P(x)=a_{0}+a_{1} x+\cdots+a_{n} x^{n}
$$

of degree at most $n$ with real coefficients and satisfying $P(-1)=P(+1)=0$. If for each $P \in \mathscr{P}_{n}$ we define

$$
\|P\|=\max _{-1 \leq x \leq 1}\left|P(x) /\left(1-x^{2}\right)^{\frac{1}{2}}\right|,
$$

$\mathscr{P}_{n}$ becomes a normed linear space. Consider a general linear functional $\omega$ on $\mathscr{P}_{n}$. There exist real numbers $\alpha_{0}, \alpha_{1}, \ldots, \alpha_{n}$ such that

$$
\omega(P)=\alpha_{0} a_{0}+\alpha_{1} a_{1}+\cdots+\alpha_{n} a_{n} \quad\left(P(x)=\sum_{\nu=0}^{n} a_{\nu} x^{\nu}\right) .
$$

We shall like to determine its norm:

$$
\|\omega\|=\sup _{\|P\| \leq 1}|\omega(P)| .
$$

For this let $\alpha$ be a real number different from zero and denote by $\mathscr{P}_{n, \alpha}$ the class of all polynomials $P \in \mathscr{P}_{n}$ for which $\omega(P)=\alpha$. If $P^{*}$ is a polynomial of smallest norm amongst all polynomials belonging to $\mathscr{P}_{n, \alpha}$ then clearly

$$
\|\omega\| \equiv\left|\omega\left(P^{*} /\left\|P^{*}\right\|\right)\right| .
$$

It is therefore of fundamental importance for us to be able to recognize polynomials $P^{*} \in \mathscr{P}_{n, \alpha}$ whose norm is the smallest. Such polynomials will hereafter be referred to as "minimal".

We shall now state three auxiliary results which are obtained by suitably modifying the proofs of (i) the lemma on p. 215 of [3], (ii) Theorem 1 on pp. 216-217 of [3], and (iii) Theorem 2 on pp. 219-220 of [3], respectively.

Lemma 1. Let $P \in \mathscr{P}_{n, \alpha}$ and let $x_{1}, x_{2}, \ldots, x_{k}$ be the roots of the equation

$$
\|P\|^{2}-\left\{P(x) /\left(1-x^{2}\right)^{\frac{1}{2}}\right\}^{2}=0
$$

lying in $(-1,1)$. Then $P$ is minimal if and only if there does not exist a polynomial 
$g \in \mathscr{P}_{n}$ such that

$$
\omega(g)=0,
$$

and the products

$$
g\left(x_{1}\right) P\left(x_{1}\right), g\left(x_{2}\right) P\left(x_{2}\right), \ldots, g\left(x_{k}\right) P\left(x_{k}\right)
$$

are all negative.

Lemma 2. Let $P \in \mathscr{P}_{n, \alpha}$ and let $x_{1}, x_{2}, \ldots, x_{k}$ be defined as above. Put

$$
F(x)=\left(1-x^{2}\right) \prod_{l=1}^{k}\left(x-x_{l}\right)
$$

and

$$
F_{l}(x)=F(x) /\left(x-x_{l}\right), \quad l=1,2, \ldots, k .
$$

Then $P$ is minimal if and only if (i) the numbers

$$
\omega\left(F_{1}\right)(-1)^{1} P\left(x_{1}\right), \ldots, \omega\left(F_{k}\right)(-1)^{k} P\left(x_{k}\right)
$$

are all of the same sign and (ii) in case $k<n-1$, we have

$$
\omega(F \psi)=0
$$

for all polynomials $\psi$ of degree at most $n-k-2$.

LEMMA 3. If the minimal polynomial is not unique then there always exists one for which equation (9) has at most $(n-3) / 2$ roots if $n$ is odd, and at most $(n-2) / 2$ roots if $n$ is even.

Let us now consider the case

$$
\omega(\varphi)=\varphi^{(j)}(t)=\left.\left(d^{j} / d x^{j}\right)[\varphi(x)]\right|_{x=t}, \quad \varphi \in \mathscr{P}_{n},
$$

where $0<j<n$ and $t$ is given. First we wish to show that in this case, equation (9) has at least $n-2$ roots in $(-1,1)$. If not, (13) which takes the form

$$
F^{(j)}(t) \psi(t)+\left(\begin{array}{l}
j \\
1
\end{array}\right) F^{(j-1)}(t) \psi^{\prime}(t)+\cdots+F(t) \psi^{(j)}(t)=0
$$

is valid for all polynomials $\psi(x)$ of degree $n-k-2(\geq 1)$ or less, where $k$ is the number of roots of $(9)$ in $(-1,1)$. Since the numbers $\psi(t), \psi^{\prime}(t), \ldots$, $\psi^{(n-k-2)}(t)$ may be chosen arbitrarily, it follows that

$$
F^{(j)}(t)=F^{(j-1)}(t)=\cdots=F(t)=0 \text { if } j \leq n-k-2
$$

whereas

$$
F^{(j)}(t)=F^{(j-1)}(t)=\cdots=F^{(j-n+k+2)}(t)=0 \quad \text { if } j>n-k-2 .
$$

Thus if $k$ were less than $n-2$ then in any case $F^{(m)}(t), F^{(m-1)}(t)$ would vanish 
for some $m(1 \leq m \leq k+2)$ which is impossible since the zeros of $F$ are real and distinct.

In view of Lemma 3 we conclude that for the functional under consideration the minimal polynomial is unique.

We are able to identify the polynomial for which $k=n-1$ and, with the help of Lemma 2, determine values of $t$ for which it is minimal. In this case, the polynomial satisfies the differential equation

$$
M^{2}-\frac{y^{2}}{1-x^{2}}=\frac{1}{4(n-1)^{2}}\left\{\left(\frac{y^{2}}{1-x^{2}}\right)^{\prime}\right\}^{2} \frac{\left(1-x^{2}\right)^{2}}{y^{2}}
$$

where $M=\max _{-1 \leq x \leq 1}\left|y /\left(1-x^{2}\right)^{\frac{1}{2}}\right|$. Solving the differential equation by separation of variables, we obtain

$$
-\frac{2}{M^{2}} \frac{y^{2}}{\left(1-x^{2}\right)}=\cos \{2(n-1) \arccos x+C\}
$$

where $C$ should be $2 k \pi$ since the polynomial vanishes at 1 . Thus

$$
y= \pm M\left(1-x^{2}\right) U_{n-2}(x),
$$

and using the fact that $\varphi^{(j)}(t)=\alpha$ we get

$$
y=\alpha\left(1-x^{2}\right) U_{n-2}(x) / P^{(j)}(t),
$$

where $P(x)=\left(1-x^{2}\right) U_{n-2}(x)$. In view of Lemma 2 , the polynomial (15) is minimal for those values of $t$ for which the corresponding numbers (12) are all of the same sign. The polynomials (10) and (11) which correspond to (15) are

$$
\begin{aligned}
& F(x)=\left(1-x^{2}\right) T_{n-1}(x) / 2^{n-2}, \\
& F_{l}(x)=\frac{\left(1-x^{2}\right) T_{n-1}(x)}{2^{n-2}\left\{x-\cos \left(l-\frac{1}{2}\right) \pi /(n-1)\right\}}, \quad l=1,2, \ldots, n-1 .
\end{aligned}
$$

The corresponding numbers (12) are

$$
\begin{aligned}
\left.\frac{-\alpha}{P^{(j)}(t) 2^{n-2}} \sqrt{1-x_{l}^{2}} \frac{d^{j}}{d x^{j}}\left\{\frac{\left(1-x^{2}\right) T_{n-1}(x)}{x-x_{l}}\right\}\right|_{x=t}, & \\
l & =1,2, \ldots, n-1,
\end{aligned}
$$

where $x_{l}=\cos \left\{\left(l-\frac{1}{2}\right) \pi /(n-1)\right\}$. Now with the help of a reasoning similar to that used by W. A. Markoff it can be proved that the numbers (16) are all of the same sign if $t$ belongs to any of the intervals

$$
\left(-\infty, \xi_{1}\right],\left[\eta_{1}, \xi_{2}\right], \ldots,\left[\eta_{n-j-1}, \xi_{n-j}\right],\left[\eta_{n-j}, \infty\right)
$$

where $\xi_{1}, \xi_{2}, \ldots, \xi_{n-j}$ and $\eta_{1}, \eta_{2}, \ldots, \eta_{n-j}$ are the roots (arranged in increasing order) of the equations

$$
\left((x+1) T_{n-1}(x)\right)^{(j)}=(x+1) T_{n-1}{ }^{(j)}(x)+j T_{n-1}{ }^{(j-1)}(x)=0
$$


and

$$
\left((x-1) T_{n-1}(x)\right)^{(j)}=(x-1) T_{n-1}^{(j)}(x)+j T_{n-1}{ }^{(j-1)}(x)=0
$$

respectively.

Using the well-known differential equation

$$
\left(x^{2}-1\right) T_{n-1}^{\prime \prime}(x)+x T_{n-1}^{\prime}(x)=(n-1)^{2} T_{n-1}(x)
$$

satisfied by $T_{n-1}(x)$ we can show that

$$
-\cos \frac{\pi}{n-1} \leq \xi_{1}<\eta_{n-j} \leq \cos \frac{\pi}{n-1} \text { if } j \geq 3
$$

and

$$
-\cos \frac{\pi}{2(n-1)} \leq \xi_{1}<\eta_{n-j} \leq \cos \frac{\pi}{2(n-1)} \quad \text { if } j=2 .
$$

We have shown, in particular, that amongst all polynomials $P \in \mathscr{P}_{n}$ for which $P^{\prime \prime}(t)=\alpha$ at a given point $t$ in

$$
[-1,-\cos (\pi / 2(n-1))]
$$

or in

$$
[\cos (\pi / 2(n-1)), 1]
$$

the polynomial (15) is of smallest norm. In other words, if $p_{n}(x)$ is a polynomial of degree $n$ such that $\left|p_{n}(x)\right| \leq \sqrt{1-x^{2}}$ for $-1 \leq x \leq 1$ then

$$
\left|p_{n}^{\prime \prime}(t)\right| \leq\left|\frac{d^{2}}{d x^{2}}\left(\left(1-x^{2}\right) U_{n-2}(x)\right)\right|_{x=t} \leq\left|\frac{d^{2}}{d x^{2}}\left(\left(1-x^{2}\right) U_{n-2}(x)\right)\right|_{x= \pm 1}
$$

if $\cos (\pi / 2(n-1)) \leq|t| \leq 1$.

For the limited purpose of getting the desired global bound (7) for the second derivative of polynomials whose graph lies in the unit disk we need not consider the (rather complicated) case $k=n-2$. We may instead complete the proof as follows.

Consider the operator $B$ which carries $p_{n}(z)=\sum_{\nu=0}^{n} a_{v} z^{\nu}$ into

$$
B\left[p_{n}(z)\right]=\lambda_{0} p_{n}(z)+\lambda_{1}(n z / 2) p_{n}^{\prime}(z) / 1 !+\lambda_{2}(n z / 2)^{2} p_{n}^{\prime \prime}(z) / 2 !
$$

where $\lambda_{0}, \lambda_{1}$, and $\lambda_{2}$ are such that all the zeros of

$$
u(z)=\lambda_{0}+\left(\begin{array}{c}
n \\
1
\end{array}\right) \lambda_{1} z+\left(\begin{array}{l}
n \\
2
\end{array}\right) \lambda_{2} z^{2}
$$

lie in the half plane $|z| \leq|z-n / 2|$. Using the fact that if all the zeros of $p_{n}(z)$ lie in $|z| \leq 1$ then [1, p. 65, see Corollary (18.3)] so do all the zeros of the polynomial $B\left[p_{n}(z)\right]$ we can prove the following result in precisely the same way as Theorem 4 of [4].

LEMMA 4. Let $q_{n}(z)=\sum_{\nu=0}^{n} b_{\nu} z^{\nu}$ with $b_{n} \neq 0$ have all its zeros in $|z| \leq 1$. 
If $p_{n}(z)$ is a polynomial of degree $n$ such that $\left|p_{n}(z)\right| \leq\left|q_{n}(z)\right|$ for $|z|=1$ then

$$
\left|B\left[p_{n}(z)\right]\right| \leq\left|B\left[q_{n}(z)\right]\right| \text { for }|z| \geq 1 .
$$

From this we can easily deduce (see [4, pp. 306-307]):

LemMa 5. Let $\tau_{n}(z)=\sum_{\nu=-n}^{n} d_{\nu} e^{i v z}$ with $d_{n} \neq 0$ be a trigonometric polynomial having all its zeros in $\operatorname{Im} z \geq 0$. If $S_{n}(z)=\sum_{\nu=-n}^{n} c_{\nu} e^{i \nu z}$ is a trigonometric polynomial of degree $n$ such that $\left|S_{n}(\theta)\right| \leq\left|\tau_{n}(\theta)\right|$ for real values of $\theta$ and the zeros of the polynomial

$$
u_{1}(z)=2 a(2 n-1) z^{2} / n-2\{a(2 n-1)+i b\} z+a n^{2}+i n b-c
$$

lie in the half plane $|z| \leq|z-n|$, then

$$
\left|a S_{n}^{\prime \prime}(\theta)+b S_{n}^{\prime}(\theta)+c S_{n}(\theta)\right| \leq\left|a \tau_{n}^{\prime \prime}(\theta)+b \tau_{n}^{\prime}(\theta)+c \tau_{n}(\theta)\right|
$$

for real $\theta$.

We observe that if $a, b, c$ are real and

$$
n b^{2}+n(2 n-1) a^{2}-2 a c(2 n-1) \geq 0,
$$

then the zeros of the polynomial $u_{1}(z)$ are of the form $n / 2 \pm i \beta$ where $\beta$ is real, i.e. they lie in the half plane $|z| \leq|z-n|$. Hence we have

LEMMA $5^{\prime}$. Let $\tau_{n}(z)$ and $S_{n}(z)$ be trigonometric polynomials as in Lemma 5. If $a, b$, and $c$ are real numbers such that (18) holds, then

$$
\left|a S_{n}^{\prime \prime}(\theta)+b S_{n}^{\prime}(\theta)+c S_{n}(\theta)\right| \leq\left|a \tau_{n}^{\prime \prime}(\theta)+b \tau_{n}^{\prime}(\theta)+c \tau_{n}(\theta)\right|
$$

for real $\boldsymbol{\theta}$.

We may use Lemma $5^{\prime}$ to show that if $p_{n}(x)$ is a polynomial of degree $n$ such that $\left|p_{n}(x)\right| \leq\left(1-x^{2}\right)^{\frac{1}{2}}$ for $-1 \leq x \leq 1$, then for $|x| \leq \cos (\pi / 2(n-1))$ we have

$$
\left|p_{n}^{\prime \prime}(x)\right| \leq\left|\frac{d^{2}}{d x^{2}}\left(\left(1-x^{2}\right) U_{n-2}(x)\right)\right|_{x= \pm 1}=\frac{2}{3}(n-1)\left(2 n^{2}-4 n+3\right) .
$$

Let $x_{0}=\cos \theta_{0}$ be an arbitrary point in

$$
[-\cos (\pi / 2(n-1)), \cos (\pi / 2(n-1))]
$$

and apply Lemma $5^{\prime}$ with $\tau_{n}(z)=e^{i(n-1) z} \sin z, \quad S_{n}(z)=p_{n}(\cos z), \quad a$ $=-\tan \theta_{0}, b=1$, and $c=0$. We obtain

$$
\left|-\frac{\sin ^{3} \theta_{0}}{\cos \theta_{0}} p_{n}^{\prime \prime}\left(\cos \theta_{0}\right)\right| \leq\left|-i(n-1) \sin \theta_{0}+\left\{(n-1)^{2}+1\right\} \frac{\sin ^{2} \theta_{0}}{\cos \theta_{0}}+\cos \theta_{0}\right|
$$

or

$$
\left|p_{n}^{\prime \prime}\left(\cos \theta_{0}\right)\right|
$$

$$
\leq \frac{1}{\left|\sin \theta_{0}\right|}\left\{n(n-1)^{2}(n-2)+3(n-1)^{2} / \sin ^{2} \theta_{0}+1 / \sin ^{4} \theta_{0}\right\} .
$$


It can be easily verified that the right-hand side of (19) does not exceed $\frac{2}{3}(n-1)\left(2 n^{2}-4 n+3\right)$ for $n \geq 2$ if $\left|\cos \theta_{0}\right| \leq \cos (\pi / 2(n-1))$ as happens to be the case. With this the proof of the following theorem is complete.

THEOREM. If $p_{n}(x)$ is a polynomial of degree $n$ such that $\left|p_{n}(x)\right| \leq\left(1-x^{2}\right)^{\frac{1}{2}}$ for $-1 \leq x \leq 1$, then

$$
\begin{aligned}
\max _{-1 \leq x \leq 1}\left|p_{n}^{\prime \prime}(x)\right| & \leq\left|\frac{d^{2}}{d x^{2}}\left(\left(1-x^{2}\right) U_{n-2}(x)\right)\right|_{x= \pm 1} \\
& =\frac{2}{3}(n-1)\left(2 n^{2}-4 n+3\right) .
\end{aligned}
$$

\section{REFERENCES}

1. M. Marden, Geometry of polynomials, 2nd ed., Math. Surveys, no. 3, Amer. Math. Soc., Providence, R.I., 1966. MR 37 \# 1562.

2. A. A. Markoff, On a problem of D. I. Mendeleev, Zap. Imp. Akad. Nauk 62 (1889), 1-24. (Russian)

3. W. A. Markoff, Uber Polynome, die in einem gegebenen Intervalle möglichst wenig von Null abweichen, Math. Ann. 77 (1916), 218-258.

4. Q. I. Rahman, Functions of exponential type, Trans. Amer. Math. Soc. 135 (1969), 295-309. MR 38 \# 1261.

5. On a problem of Turán about polynomials with curved majorants, Trans. Amer. Math. Soc. 163 (1972), $447-455$. MR 45 \#3656.

Département de Mathématique, Université de Montréal, Montreal, QuÉbec, Canada 